\title{
Is Measuring Risk Marker Progression Useful for Cardiovascular Disease Prediction?
}

\author{
Akira Fujiyoshi $^{\mathrm{a}}$ Maryam Zaid $^{\mathrm{b}}$ Emma Barinas-Mitchell ${ }^{\mathrm{c}}$ \\ ${ }^{a}$ Department of Hygiene, Wakayama Medical University, Wakayama, Japan; ${ }^{b}$ Department of Epidemiology, School \\ of Public Health, Fudan University, Shanghai, China; 'Department of Epidemiology, Graduate School of Public \\ Health, University of Pittsburgh, Pittsburgh, PA, USA
}

Dear Editor,

There has been much debate on whether measuring the progression of carotid intima-media thickness (cIMT) adds further predictive information for future CVD events than simply utilizing single baseline measures. Pertinent studies have been conducted in various settings, including pooled projects of observational studies (general populations [1], diabetics [2], and high-risk individuals [3], see Table 1). Many, but not all, of these observational studies have reported null associations between progression, defined as the difference in cIMT measured at 2 separate time points, and risk of CVD events. In contrast, a recently published meta-analysis of clinical trial data [4] showed a significant association of cIMT progression with risk of CVD events with smaller cIMT progression per year associated with lower risk [4]. In the face of this apparent inconsistency, the authors of the meta-analysis stated that the null associations previously reported "could be explained by the challenges of precisely estimating cIMT progression in individuals over time," and that focusing "on groups of patients in RCTs is better suited" for assessing the value of cIMT progression [4].

karger@karger.com

(c) 2021 S. Karger AG, Basel

www.karger.com/ced

Karger"
Certainly, a study with low reproducibility of cIMT measurements is likely to result in a null association. However, there is no clear reason why RCTs are superior to observational studies with respect to precision of cIMT measurement. In a meta-analysis or pooled analysis of individual participant data, heterogeneity among assessed studies may result in failure to capture the true association [5], although we have no reason to believe that heterogeneity is always less in RCTs than in observational studies. As for other possibilities, a small true change in cIMT over time, due to either a short duration between the first and second measurement or to the use of low-risk versus high-risk populations, will likely result in a low signal-to-noise ratio [6]. This possibility, however, seems unlikely to explain the inconsistent results on the association between cIMT progression and CVD risk at least among recent studies because the average duration between cIMT measurement and the annualized change in cIMT were similar across those studies (Table 1).

We would like to raise another possibility that differences in statistical models used among the studies may, at least in part, explain the inconsistency. In particular, most [1-3], but not all [7], studies reporting null associations adopted a model that simultaneously included "progres-

Correspondence to:

Akira Fujiyoshi, afujiy@ wakayama-med.ac.jp 


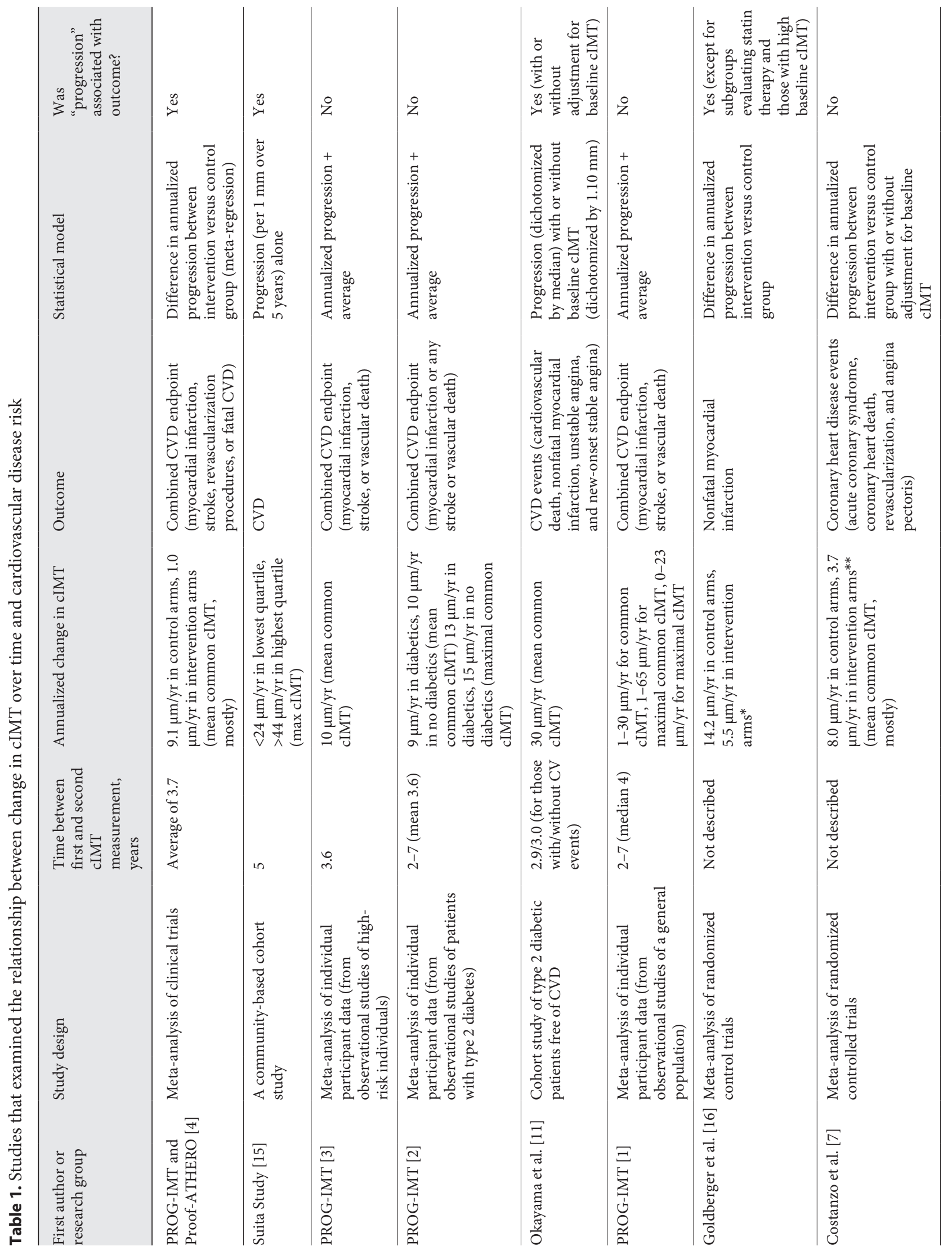




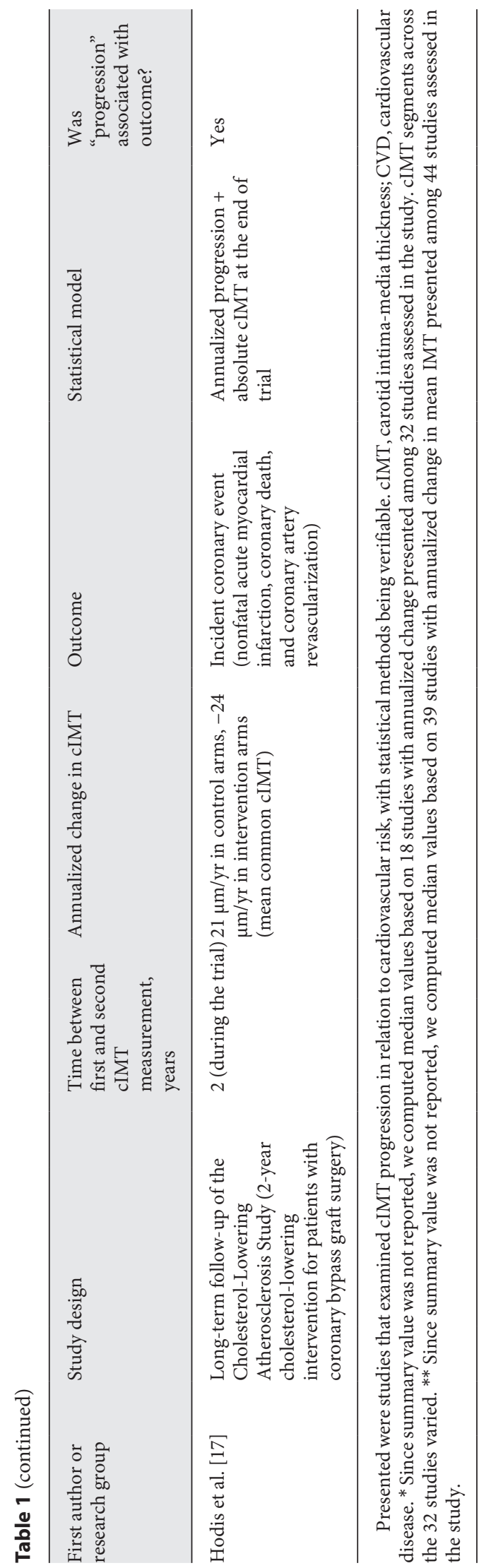

sion" and "average" of the 2 cIMT values assessed at 2 separate time points ("progression-average model"), whereas many studies reporting a significant positive association did not adopt such a model, including the aforementioned meta-analysis (Table 1).

To illustrate why the "progression-average model" matters, suppose a simple regression model: CVD risk = beta $1 \times R F 1+$ beta $2 \times R F 2$, where RF denotes a risk factor or marker. If a risk factor assessed at time points 1 and 2 (RF1 and RF2, respectively) has a similar strength of association with CVD risk in the model, then the regression coefficient of progression (RF2 - RF1) in the "progression-average model" is determined to be small, close to zero, because the coefficient is mathematically equivalent to (beta2 - beta1)/2 [8]. A near-zero regression coefficient for progression obtained from the "progression-average model" is likely to be statistically nonsignificant. In fact, even for a conventional risk factor such as systolic blood pressure, the association between progression and CVD risk can be either null or positive depending on the model used: a null (i.e., nonsignificant) association observed in the "progression-average model" flipped to be significantly positive when based on models without the average term $[9,10]$. As such, previously reported "null associations" may be just artifacts derived from the "progression-average model." Thus, we are concerned that the null findings, based on the "progression-average model," may provide a biased impression that the second time measurement of the risk marker has no value, which indeed means, when based on the simple model, that the second time measurement predicts CVD events to a similar degree to the first measurement.

What would be the "correct model" then? In our opinion, there is no single "correct model" that is suitable for every case. The answer also depends on the aim of a study. If the aim is to examine the predictive property of RF2 for CVD risk, then stratification according to cIMT levels at baseline (RF1) and at second measure (RF2) to assess how CVD risk differs across the strata would be a simple and easy-to-understand model $[11,12]$. Alternatively, the "simple regression model" described above may be acceptable if correlation between RF1 and RF2 is not a concern. When the aim is to examine the additive value of the repeated measurement(s) for CVD prediction, multiple options without using the "progression" variable have been proposed such as use of the average [13], cumulative value or trajectory patterns of risk factor/marker over time [14].

To conclude, in order to assess the usefulness of risk factors/markers assessed at different time points in pre- 
dicting CVD events, one needs to carefully choose an appropriate statistical model. Otherwise, this debate will not be settled, and we will continue without substantial progress in this dispute.

\section{Conflict of Interest Statement}

The authors have no conflicts of interest to declare.

\section{Funding Sources}

This work has been conducted without any funding source.

\section{Author Contributions}

A.F. conceived and drafted the first version of the manuscript and revised. M.Z. and E.B.-M. assisted collecting published articles, critically reviewed earlier versions of the draft, and contributed to revision. All the authors approved the final version.

\section{References}

1 Lorenz MW, Polak JF, Kavousi M, Mathiesen EB, Völzke H, Tuomainen TP, et al. Carotid intima-media thickness progression to predict cardiovascular events in the general population (the PROG-IMT collaborative project): a meta-analysis of individual participant data. Lancet. 2012;379(9831):2053-62.

2 Lorenz MW, Price JF, Robertson C, Bots ML, Polak JF, Poppert H, et al. Carotid intima-media thickness progression and risk of vascular events in people with diabetes: results from the PROG-IMT collaboration. Diates Care. 2015;38(10):1921-9.

3 Lorenz MW, Gao L, Ziegelbauer K, Norata GD, Empana JP, Schmidtmann I, et al. Predictive value for cardiovascular events of common carotid intima media thickness and its rate of change in individuals at high cardiovascular risk - results from the PROG-IMT collaboration. PLoS One. 2018;13(4): e0191172.

4 Willeit P, Tschiderer L, Allara E, Reuber K, Seekircher L, Gao L, et al. Carotid intima-media thickness progression as surrogate marker for cardiovascular risk: meta-analysis of 119 clinical trials involving 100667 patients. Circulation. 2020;142(7):621-42.

5 Taylor AJ, Bots ML, Kastelein JJ. Vascular disease: meta-regression of CIMT trials-data in, garbage out. Nat Rev Cardiol. 2011;8(3):12830.

6 Nambi V, Pedroza C, Kao LS. Carotid intimamedia thickness and cardiovascular events. Lancet. 2012;379(9831):2028-30.
7 Costanzo P, Perrone-Filardi P, Vassallo E, Paolillo S, Cesarano P, Brevetti G, et al. Does carotid intima-media thickness regression predict reduction of cardiovascular events? A meta-analysis of 41 randomized trials. J Am Coll Cardiol. 2010;56(24):2006-20.

8 Fujiyoshi A. "Progression" of a risk factor on cardiovascular outcome: a valuable point, a questionable interpretation. Eur J Prev Cardiol. 2020;27(3):231-3.

9 Bahls M, Lorenz MW, Dörr M, Gao L, Kitagawa K, Tuomainen TP, et al. Progression of conventional cardiovascular risk factors and vascular disease risk in individuals: insights from the PROG-IMT consortium. Eur J Prev Cardiol. 2020;27(3):234-43.

10 Menotti A, Blackburn H, Kromhout D, Nissinen A, Fidanza F, Giampaoli S, et al. Changes in population cholesterol levels and coronary heart disease deaths in seven countries. Eur Heart J. 1997;18(4):566-71.

11 Okayama KI, Mita T, Gosho M, Yamamoto R, Yoshida M, Kanazawa A, et al. Carotid intima-media thickness progression predicts cardiovascular events in Japanese patients with type 2 diabetes. Diabetes Res Clin Pract. 2013; 101(3):286-92.

12 Allen N, Berry JD, Ning H, Van Horn L, Dyer A, Lloyd-Jones DM. Impact of blood pressure and blood pressure change during middle age on the remaining lifetime risk for cardiovascular disease: the cardiovascular lifetime risk pooling project. Circulation. 2012;125(1):3744.
13 Cho IJ, Sung JM, Chang HJ, Chung N, Kim HC. Incremental value of repeated risk factor measurements for cardiovascular disease prediction in middle-aged Korean adults: results from the NHIS-HEALS (national health insurance system-national health screening cohort). Circ Cardiovasc Qual Outcomes. 2017; 10(11):e004197.

14 Nuotio J, Suvila K, Cheng S, Langén V, Niiranen T. Longitudinal blood pressure patterns and cardiovascular disease risk. Ann Med. 2020 May-Jun;52(3-4):43-54.

15 Kokubo Y, Watanabe M, Higashiyama A, Nakao YM, Nakamura F, Miyamoto Y. Impact of intima-media thickness progression in the common carotid arteries on the risk of incident cardiovascular disease in the suita study. J Am Heart Assoc. 2018;7(11):e007720.

16 Goldberger ZD, Valle JA, Dandekar VK, Chan PS, Ko DT, Nallamothu BK. Are changes in carotid intima-media thickness related to risk of nonfatal myocardial infarction? A critical review and meta-regression analysis. Am Heart J. 2010;160(4):701-14.

17 Hodis HN, Mack WJ, LaBree L, Selzer RH, Liu $\mathrm{CR}$, Liu $\mathrm{CH}$, et al. The role of carotid arterial intima-media thickness in predicting clinical coronary events. Ann Intern Med. 1998; 128(4):262-9. 